\title{
Elexacaftor/Tezacaftor/lvacaftor alters branching morphogenesis of the mouse embryonic lung
}

Mickaël Lhuillier ${ }^{1 \#}$, Laura Aoust ${ }^{1,2}$, Elise Dreano ${ }^{1}$, Marie-Laure Franco-Montoya ${ }^{3}$, Kim Landry-Truchon ${ }^{4}$, Nicolas Houde ${ }^{4}$, Stéphanie Chhun ${ }^{5,6}$, Alexandre Hinzpeter ${ }^{1}$, Aleksander Edelman ${ }^{1}$, Christophe Delacourt ${ }^{1,6,7}$, Lucie Jeannotte ${ }^{4,8}$, Isabelle SermetGaudelus $^{1,2,6,7,9 *}$, Alice Hadchouel ${ }^{1,6,7 *}$.

${ }^{1}$ INSERM U1151, Institut Necker Enfants Malades, Paris, France.

${ }^{2}$ Centre de Références Maladies Rares, Mucoviscidose et Maladies apparentées, Hôpital Necker Enfants Malades, Assistance Publique-Hôpitaux de Paris (AP-HP)Centre, Paris, France.

${ }^{3}$ INSERM U955, Institut Mondor de Recherche Biomédicale, Créteil, France.

${ }^{4}$ Centre de recherche sur le cancer de l'Université Laval, Centre de recherche du CHU de Québec-Université Laval (Oncology Axis), Québec, Canada.

${ }^{5}$ Laboratoire d'immunologie biologique, Hôpital Necker Enfants Malades, Paris, France.

${ }^{6}$ Université de Paris, Paris, France.

${ }^{7}$ Service de Pneumologie Pédiatrique, Centre de Référence pour les Maladies Respiratoires Rares de l'Enfant, Hôpital Universitaire Necker-Enfants Malades, Paris, France.

${ }^{8}$ Department of Molecular Biology, Medical Biochemistry \& Pathology, Université Laval, Québec, Canada.

${ }^{9}$ European Respiratory Network for Lung Disease.

* The co-last two authors contributed equally.

\# Present address: Centre de recherche sur le cancer de l'Université Laval, Centre de recherche du CHU de Québec-Université Laval (Oncology Axis), Québec, Canada. 
Corresponding author: isabelle Sermet-Gaudelus, Isabelle.sermet@aphp.fr;

INSERM U1151, Institut Necker Enfants Malades, Université de Paris, Hôpital Necker Enfants Malades, 149 rue de Sévres, 75015 Paris, France

\section{Take home message}

The triple association Elexacaftor/Tezacaftor/lvacaftor alters lung branching morphogenesis of murine F508Del heterozygous embryos. Those results argue for a close monitoring of pregnancies in women treated with this combo-therapy. 


\section{Abstract}

\section{Introduction}

CFTR modulators triple combo-therapy Elexacaftor/Tezacaftor/lvacaftor (ETI) has proven to clinically benefit homozygous and heterozygous F508del patients. As a result, an increasing number of pregnancies is expected. Studies of the potential impact of these modulators on the development of the foetus are mandatory.

\section{Materials}

We used the early mouse embryonic lung organ culture model to analyse ex vivo the lung branching process and the relative expression of Fgf10, Fgfr2IIlb, Shh, and Hhip development regulator genes in different conditions: standard culture medium, treatment with ETI or with Forskolin \pm Inh172. Development of lung branching and distal bud caliber were evaluated in lung explants from heterozygous F508del Cftr $^{\mathrm{tm} 1 \mathrm{Eur} /+}$ and control $\mathrm{Cftr}^{\mathrm{tm} 1 \mathrm{Eur}+\mathrm{H}_{+}}$(WT) mouse embryos at E12.5 during pseudoglandular stage.

\section{Results}

Exposure to ETI of the Cftr ${ }^{\mathrm{tm} 1 \mathrm{Eur} /+}$ and WT lung explants induced a significant decrease in lung branching after $48 \mathrm{~h}$ culture and the percentage of terminal bud dilations was significantly increased. These results were recapitulated by cAMPdependent CFTR continuous activation by Forskolin and reversed by addition of Inh172.

ETI induced a significant decrease in Fgf10, Fgfr2IIlb, Shh and Hhip expression in lung explants of both E12.5 $\mathrm{Cftr}^{\mathrm{tm} 1 \mathrm{Eur} / \mathrm{+}}$ and WT embryos treated with ETI for $72 \mathrm{~h}$.

\section{Conclusion}


Our results provide evidence that the triple association Elexacaftor/Tezacaftor/lvacaftor alters lung branching morphogenesis of WT and heterozygous F508del mouse embryos during the pseudo-glandular stage. Those results argue for a close monitoring of pregnancies in patients treated with these drugs.

\section{Plain Language}

\section{Introduction}

The triple combo-therapy Elexacaftor/Tezacaftor/lvacaftor (ETI) improves homozygous and heterozygous F508del patients. As a result, an increasing number of pregnancies is expected. Studies of this treatment on the development of the foetus are lacking. We incubated lungs of murine foetus not carrying CFTR mutation or F508del heterozygous. We show that ETI induces significant defect of lung development and the formation of cysts. These results are at least partly due to CFTR activation. Those results argue for a close monitoring of pregnancies in patients treated with these drugs. 
bioRxiv preprint doi: https://doi.org/10.1101/2021.11.01.466814; this version posted November 4, 2021. The copyright holder for this preprint (which was not certified by peer review) is the author/funder, who has granted bioRxiv a license to display the preprint in perpetuity. It is made available under aCC-BY-NC-ND 4.0 International license. 


\section{Introduction}

Cystic Fibrosis (CF) is the most common monogenic autosomal recessive disease of people of Caucasian descent with an estimated incidence between 1/3000 to $1 / 6000$ births in European populations [1, 2]. CF is caused by mutations in the Cystic Fibrosis Transmembrane Conductance Regulator (CFTR) gene, which leads to defective expression and/or function of the CFTR protein. The most frequent mutation in the Caucasian population is the Phe508del mutation (F508del thereafter). In the respiratory tract, CFTR dysfunction is associated with altered mucus clearance, chronic lung inflammation, recurrent respiratory infections, and ultimately respiratory failure [3].

CFTR modulators triple combo-therapy Elexacaftor/Tezacaftor/lvacaftor (ETI hereafter) has proven to clinically benefit homozygous and heterozygous F508del patients [4-7] and to improve CF patient's fertility. As a result, young women with CF are more likely to become pregnant and give birth [8]. With the lack of evidence of safety during pregnancy, studies aiming to understand the potential impact of these modulators on the development of the foetus are mandatory. Until now a limited number of pregnancies upon CFTR modulators, (mainly Ivacaftor or LumacaftorIvacaftor) [9-14], and very few upon ETI, occurred and showed no alarming signals [15].

In the human developing lung, CFTR expression is detected from the $12^{\text {th }}$ week of gestation [16] and is involved in lung fluid secretion and airway budding [17]. This is suggested by observations in pigs, which showed that invalidation of CFTR alters pseudo-glandular development inducing a significant reduction of the caliber of the trachea and the proximal bronchi together with the formation of hypo-distended airway budding [18]. In animal reproductive models, Ivacaftor, Tezacaftor and 
Elexacaftor cross the placenta, and the mean foetal lung concentration ratio is very high, around $200 \%$ of the plasma, much higher than into brain or cerebrospinal fluid $[13,19]$

We asked whether ETI might impact foetal lung organogenesis, and more specifically if it might affect lung branching and distal bud formation in mice carrying the +/F508del heterozygous genotype. To reach this aim, we investigated the effect of ETI on the development of embryonic lung during pseudo-glandular stage from heterozygous +/F508del Cftr mice and more specifically lung branching and distal buds' formation. We used the early mouse embryonic lung organ culture model to analyse ex vivo the lung branching process and further dissect the molecular mechanisms involved.

\section{Material and methods}

\section{Animal experiments}

Experiments were performed according to the guidelines of European directive 2010/63/UE with the approbation of ethics committee for animal experiments of Paris Descartes University (CEEA n`34).

C57BI6j male and female wild type (WT) mice were obtained from Charles River (Saint-Germain-Nuelles, France). Heterozygous male and female mice carrying the F508del mutation in the Cftr gene (Cftr ${ }^{\text {t1Eur }}$ mouse) [20] were obtained from CNRSUPS44 "Transgenesis and storage of animal models" at Orléans (France). Age of the embryos was estimated by considering the morning of the day of vaginal plug as the first half day of pregnancy (E0.5). Pregnant female mice were killed by cervical dislocation and lungs were collected from embryos after 12 days of gestation (E12.5) 
corresponding to the human pseudo-glandular stage (5 to 17 weeks of gestation).

Tail tissue was collected for genotyping by PCR.

\section{Mouse embryo lung explant cultures}

Lung explants were collected from E12.5 mouse embryos and cultured in serum-free Dulbecco's Modified Eagle Medium (DMEM/F12 (Gibco 31331093, Thermofischer Scientific, Illkirch, France) for $72 \mathrm{~h}$ on porous membranes (Whatman ${ }^{\circledR}$ Nucleopore ${ }^{\mathrm{TM}}$ Track-Etched Membranes WHA150446, Sigma Aldrich, St. Quentin Fallavier, France) in 4-well plates [21]. Pictures of the explants were taken at baseline (T0) and after 24 hours (T24), 48 hours (T48) and 72 hours (T72) of culture. The number of terminal buds at the periphery of each sample was counted and the branching growth was estimated by the relative ratio (Branches T24, T48 or T72-Branches T0) $x$ 100/Branches T0 as previous described by Boucherat et al [22]. It was compared to the DMEM/F12 control group, at each time point, in percentage of variation from this reference group.

The diameter of the terminal buds of the explants was measured after $72 \mathrm{~h}$ of culture using the ImageJ software. A terminal bud dilation was defined by a diameter of the terminal bud greater than two standard deviations (SD) from the mean diameter of the DMEM/F12 control group. The results were reported as the ratio of the number of dilations in the treated group to that reported in the DMEM/F12 control group.

To modulate CFTR protein activity, culture medium was supplemented with CFTR modulators combination at the following concentrations:

- Elexacaftor (S8851, Selleckchem, Souffelweyersheim, France) diluted in DMSO

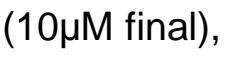

- Tezacaftor (S7059, Selleckchem, Souffelweyersheim, France) diluted in DMSO $(10 \mu \mathrm{M}$ final), 
- Ivacaftor (S1174, Selleckchem, Souffelweyersheim, France) diluted in DMSO (100nM final),

- Forskolin (F6886, St. Quentin Fallavier, France) diluted in EtOH 100\% (5 $\mu \mathrm{M}$ final) was used to increase cAMP intracellular content and activate CFTR phosphorylation, - CFTR inhibitor Inh-172 (C2992, Sigma Aldrich, St. Quentin Fallavier, France) diluted in DMSO (5 $\mu \mathrm{M}$ final) was used to specifically inhibit CFTR.

\section{Real-time quantitative PCR analysis}

Expression of Cftr expression was monitored during mouse lung development by RTqPCR assays during gestation at E12.5, E15.5 and E18.5 and after birth at P0, P7, P15 and P30 in WT specimens (See Online supplement).

RT-qPCR expression analyses of Fgf10, Fgfr2IIIb, Shh, and Hhip were performed with RNA from E12.5 lung explants snap-frozen after $72 \mathrm{~h}$ of culture (See Online supplement).

\section{Statistical analyses}

Data were expressed as mean and Standard Error of the Mean (s.e.m). They were analysed with repeated measures ANOVA, and two groups comparison were performed by Mann-Whitney $U$ test using GraphPad Prism software (GraphPad Software INC, San Diego, CA). A significance level inferior to $5 \%(p<0.05)$ was considered statistically significant.

\section{Results}

\section{The F508del mutation does not alter lung branching morphogenesis in mice}

In WT mice, Cftr expression was detected throughout lung formation from E12.5 onwards with a maximum level at E18.5 just before birth (Figure S1). 
There was no significant difference between lung explants from WT Cftr ${ }^{+/}$, F508del heterozygous $C \mathrm{ftr}^{\mathrm{tm} 1 \text { Eur/+ }}$ or F508del homozygous $\mathrm{Cftr}^{\mathrm{tm} 1 \text { Eur/tm1Eur }}$ embryos at E12.5 neither for lung branching nor for terminal buds' dilations (Figure 1, Table 1). Lung branching increased similarly in the three groups at each time point, up to $302 \% \pm$ $27 \%$ in the WT group, $263 \% \pm 15 \%$ in the Cftr $^{\text {tm } 1 \text { Eur/+ }}$ group, and $281 \% \pm 20 \%$ in the Cftr $^{\text {tm1Eur/m1Eur }}$ group at T72 (Not Significant (NS)). Moreover, there was no significant difference in the percentage of terminal buds' dilations after $72 \mathrm{~h}$ of culture between the three groups. Those results demonstrate that carrying one or two Cftr F508del variant alleles does not have major impact on lung branching morphogenesis.

\section{CFTR gain of function induced by ETI affects lung development in F508del heterozygous Cftr $^{\text {tm1Eur/+ }}$ mice}

We investigated the effect of 3-day exposure to ETI on branching morphogenesis in lung explants from $\mathrm{Cftr}^{\mathrm{tm} 1 \mathrm{Eur} /+}$ embryos collected at E12.5. We first validated that per se DMSO, used as vehicle for ETI, has no effect on lung branching in culture when compared to media (Figure S2, Table S2).

ETI incubation did not induce cytotoxicity as shown by the normal aspect of all lung explants and the easy distinction of the epithelial and mesenchymal layers. As shown in Figure 2 and Table 1, there was a significant decrease in lung branching after 48h and $72 \mathrm{~h}$ of culture for the $\mathrm{Cftr}^{\mathrm{tm} 1 \mathrm{Eur} /+}$ lung explants treated with ETI as compared to the control group incubated with media. The mean lung branching of the ETI group was decreased by $51 \% \pm 8 \%$ at T24 (NS), $72 \% \pm 3 \%$ at T48 $(\mathrm{p}<0.0001)$ and $78 \% \pm$ $2 \%$ at T72 $(p<0.0001)$. The percentage of terminal buds' dilations of $\mathrm{Cftr}^{\mathrm{tm} 1 \text { Eur/+ }}$ lung explants was significantly increased by 5.3 -fold $( \pm 0.3)$ after 72 hours $(p<0.001)$. 
Altogether, these results suggest that acute ETI exposure of the lung at the pseudoglandular stage negatively impacts on lung branching, and results in the formation of abnormal terminal dilations.

\section{ETI affects lung branching morphogenesis development in WT mice.}

To investigate the mechanisms underlying this pathogenic effect, we then asked whether the effect of ETI on lung branching might be related to the F508del mutation of Cftr. To this aim, we studied the effect of ETI on WT mouse lung explants at T24, T48 and T72 (Figure 3, Table 1). Lung branching was decreased upon incubation with ETI by $61 \% \pm 12 \%$ at T24 (NS), $25 \% \pm 6 \%$ at T48 $(\mathrm{p}<0.0001)$ and $21 \% \pm 4 \%$ at T72 $(p<0.0001)$ in comparison to the media control group. Similarly, there was a significant increase in the percentage of terminal buds' dilations by 5.7 -fold $( \pm 0.3)$ after $72 \mathrm{~h}$ of culture for the ETI group when compared to the media control group $(p=0.004)$. These changes were not significantly different from those observed in the Cftr $^{\mathrm{tm} 1 \mathrm{Eur} /+}$ explants treated with ETI (Table 1) suggesting that the ETI triple combination affects lung branching morphogenesis independently of the presence of a F508del Cftr mutation.

\section{CFTR activation mediated by cAMP affects lung development in mice}

As the mechanism of action of these drugs relies on the restoration of CFTR activity, we investigated whether the lung development defects observed after ETI exposure were associated to an increase in CFTR activity. To mimic this, we exposed lung embryos to the adenylyl cyclase activator Forskolin, which activates CFTR via PKAdependent phosphorylation. We also checked the specificity of Forskolin action on CFTR by adding the CFTR specific inhibitor Inh172 in the culture medium (Figure 4, Table 1). 
We verified that per se $\mathrm{EtOH}$, the vehicle of Forskolin, had no effect on lung branching compared to media (Figure S2, Table S2). We observed a significant decrease in lung branching after incubation with Forskolin of the lung explants at all time points tested. The mean lung branching was decreased by $67 \% \pm 4 \%$ at T24 $(p=0.0001), 36 \% \pm 4 \%$ at T48 $(p<0.0001)$ and $38 \% \pm 4 \%$ at T72 $(p<0.0001)$ in the Forskolin-treated group compared to the media control group. There was also a significant increase of the mean percentage of terminal buds' dilations by 2.3-fold $( \pm 0.3)$ in the Forskolin-treated group $(p<0.05)$.

Addition of Inh172 in combination with Forskolin to the culture medium rescued the lung branching phenotype and the mean of percentage of terminal buds' dilations when compared to the media control group (Figure 4, Table 1). Altogether, these results show that the lung branching morphogenesis defects induced by $\mathrm{ETI}$ are recapitulated by the cAMP-dependent CFTR continuous activation.

\section{ETI modulates the expression of genes involved in foetal lung development in} mice.

We studied whether ETI modifies the expression of genes known to participate in fetal mouse lung development (Figure 5, Table 2). To do so, we assessed the expression of (i) Fibroblast Growth Factor 10 (FGF10), a chemoattractant crucial to lung branching and produced by clustered mesenchymal cells at sites where epithelial lung buds will elongate [23]; (ii) FGFR2IIIb, the FGF10 receptor on epithelial lung cells; (iii) Sonic Hedgehog $(\mathrm{SHH})$ and Hedgehog-interacting protein (HHIP). SHH restricts FGF10 expression to the distal tips of the lung buds and, together with HHIP, inhibits FGF10 expression in the inter-bud regions, allowing localized new bud outgrowth [24]. 
RT-qPCR assays revealed that there was a significant decrease in Fgf10, Fgfr2IIlb, Shh and Hhip expression in lung explants from both $\mathrm{E} 12.5 \mathrm{Cftr}^{\mathrm{tm} 1 \mathrm{Eur} /+}$ and WT embryos treated by ETI when compared to the media-treated group (Figure 5a, Table 2).

The same expression profile was observed for Fgf10 and Hhip in lung explants of WT embryos cultured in presence of Forskolin compared to the DMEM/F12 group $(p=0.03)$ (Figure $5 b$, Table 2$)$. This effect was partially reversed by the addition of Inh172. This contrasted with the increased expression of Fgfr2Illb and Shh expression after incubation with Forskolin when compared to the media group $(p=0.03$ each). The expression of these two genes was partially restored to the control group level in the lung of WT embryos cultured with Forskolin+Inh172 but the difference was not statistically significant.

\section{Discussion}

Our results provide evidence that the triple association of Elexacaftor/Tezacaftor/Ivacaftor alters lung morphogenesis of WT and heterozygous F508del mouse embryos at the pseudo-glandular stage by inducing lung branching defects and abnormal terminal bud's dilation. This is related, at least partially, to CFTR activation as a similar morphological pattern is observed in lung from WT embryos cultured with the cAMP activator Forskolin, and this phenotype is partially reversed with the addition of the CFTR specific inhibitor, Inh172. These morphological changes are associated with decreased transcript levels of genes involved in mouse foetal lung development, including the growth factor Fgf10 and its receptor Fgfr2IIlb, the ligand Shh and its effector Hhip. These observations made in a 
murine model suggest that ETI treatment may alter lung morphogenesis and induce the development of cystic lesions by inhibiting FGF10 signalling.

CFTR expression is spatially and temporally regulated in the human foetal lung [16, 25-30]. CFTR transcripts are detected strongly from the $12^{\text {th }}$ week of pregnancy at the pseudo-glandular stage, the period when the conducting airways and initial acinar framework develop, with a progressive increase up to the $24^{\text {th }}$ week followed by a very low expression after birth. Similarly to the human lung, we found in our mouse model, a gradual increase of Cftr transcript levels from the pseudo-glandular stage at E12.5, up to the canalicular stage at E18.5, further followed by a decrease from birth onwards. During lung development, CFTR is localized in the epithelium of the small airways [31], and is involved in foetal lung liquid secretion and lung organogenesis, possibly participating to the mechanico-sensory process regulating $W n t / \beta$-catenin signalling [29]. Its specific inhibition in human foetal lung explants significantly decreases chloride and fluid active secretion [17]. However, defective expression of CFTR is not associated with abnormal lung organogenesis as the CF babies do not have abnormal lung airways and we did not observe a significant modification in lung branching and terminal bud dilations between embryos carrying the WT $\left(\mathrm{Cftr}^{+/+}\right)$, the heterozygous $\mathrm{Cftr}^{\mathrm{tm} 1 \mathrm{Eur} /+}$ and the $\mathrm{Cftr}^{\mathrm{tm} 1 \text { Eur/tm1Eur }}$ genotypes.

In this study, we show that the pharmacological activation of CFTR at the pseudoglandular stage with ETI induces lung morphogenesis alterations characterized by major defects in lung branching and terminal bud dilation. We provide evidence that these defects are due to CFTR activation because similar defects are observed in WT embryonic lung explants treated with Forskolin, a phenotype reversed by the addition of the CFTR inhibitor Inh172. These results recall those obtained in the kidney epithelium where Forskolin increases the size and the number of cysts formed 
by Madin-Darby canine kidney cells expressing WT human CFTR, a pattern which was totally reversed by addition of CFTR Inh172 [32, 33]. This suggests that pharmacological activation of WT CFTR may contribute to the formation of epithelial cystic structures by increasing fluid secretion. In the context of F508del heterozygosity, this would combine an excessive and continuous activation of both the WT CFTR and of the corrected F508del CFTR.

Importantly, we observed that ETI consistently decreases transcript levels of both Fgf10 and its receptor in epithelial cells Fgfr2IIlb. In the human lung, defective FGF10 and FGFR2IIIb expression are associated with defective lung branching and cyst-like terminal buds' dilations [34]. Decrease in Fgf10 expression was also detected after Forskolin exposure, and was partially rescued by Inh172, showing that this modification was related, directly or indirectly, to CFTR activation. In contrast, Fgfr2IIIb and Shh were decreased upon ETI exposure but were increased after Forskolin exposure. $\mathrm{SHH}$ signalling pathway is known to occur as a negative feedback mechanism during lung branching morphogenesis in mouse by inhibiting the local FGF10 signal [35]. The combined decreases in Fgf10 expression and of its receptor after ETI exposure should ultimately lead to the total arrest of lung branching, whereas for Forskolin, increase in Shh might inhibit FGF10 signal competing with the remaining Fgfr2lllb expression, which would maintain a partial lung branching. The decreased Shh expression observed in the lung explants of WT and heterozygous F508del Cftr embryos treated with ETI suggests that the arrest of lung branching seen with these lung explants could implicate additional molecular mechanisms that are still unknown.

The molecular mechanisms of action of the ETI drugs remain unknown. Our results suggest an interaction between CFTR activation mediated by ETI modulators and the 
FGF10-FGFR2IIlb signalling pathway. Further investigations are necessary to elucidate more precisely the molecular mechanisms involved in these developmental defects, keeping in mind that modulation of the FGF10 pathway may not only affect lung but also other organ morphogenesis, such as kidney.

There are many pregnancies expected to occur upon ETI treatment. Indeed, the improving effect of this therapy is so strong that more and more CF young women will undertake pregnancies. However, so far there are very few data on the human foetal and neonatal outcomes for pregnant women treated by Elexacaftor, Tezacaftor or Ivacaftor. In this context, although there are interspecies differences in the structural and functional features of the placenta, animal studies are helpful in predicting drug toxicity during pregnancy [15]. No teratogenicity was observed when pregnant rats and mice were challenged with at least 3 times the maximum recommended human dose of each drug independently although drug transfer across the placenta was observed [13]._However, even though those studies are able to detect obvious macroscopic agenesis or aplasia, they do not evaluate more subtle organogenesis defect such as formation of the bronchial tree.

In human, there are few pharmacological data about CFTR modulators during pregnancy. Trimble et al showed a significant higher concentration of Lumacaftor in the cord blood compared to the maternal plasma concentrations and an equivalent concentration of Ivacaftor [13]. Placental transfer of Ivacaftor was also observed in recent studies in CF animal models $[19,36,37]$ and it is estimated to be around $40 \%$ $\pm 20 \%$. The mean foetal lung concentration ratio was very high, around $200 \%$ of the plasma, much higher than into brain or cerebrospinal fluid (CSF) [19].

Currently, it is recommended that CF pregnant women stop their medication during pregnancy and breastfeeding, based on limited human data. Our observation of lung 
branching arrest and cyst formation in ETI exposed explants call for caution.

Development of other organs such as kidney might be also affected as FGF10 activates several intracellular signalling cascades, resulting in cell proliferation and differentiation [38]. This strengthens the current recommendations, or at least argues for a close monitoring of those pregnancies.

\section{Support statement}

This study was funded by the Legs Poix 2017 - Chancellerie des Universités de Paris (to AH), and by the Cancer Research Society (\#22054 and 24217 to L.J.).

L. Aoust was funded by le Fonds de Recherche en Santé Respiratoire et la Fondation du Souffle (FR2017).

\section{Acknowledgements}

We thank the Animal core facility of Institut Necker Enfants Malades. 


\section{References}

1. Southern KW, Munck A, Pollitt R, et al. A survey of newborn screening for cystic fibrosis in Europe. J. Cyst. Fibros. 2007; 6: 57-65.

2. Scotet V, L'Hostis C, Férec C. The Changing Epidemiology of Cystic Fibrosis: Incidence, Survival and Impact of the CFTR Gene Discovery. Genes 2020; 11: 589.

3. Elborn JS. Cystic fibrosis. The Lancet 2016; 388: 2519-2531.

4. Davies JC, Moskowitz SM, Brown C, et al. VX-659-Tezacaftor-Ivacaftor in Patients with Cystic Fibrosis and One or Two Phe508del Alleles. N. Engl. J. Med. 2018; 379: 1599-1611.

5. Keating D, Marigowda G, Burr L, et al. VX-445-Tezacaftor-Ivacaftor in Patients with Cystic Fibrosis and One or Two Phe508del Alleles. N. Engl. J. Med. 2018; 379: 1612-1620.

6. Middleton PG, Mall MA, Dřevínek P, et al. Elexacaftor-Tezacaftor-Ivacaftor for Cystic Fibrosis with a Single Phe508del Allele. N. Engl. J. Med. 2019; 381: 1809-1819.

7. Taylor-Cousar JL, Mall MA, Ramsey BW, et al. Clinical development of triple-combination CFTR modulators for cystic fibrosis patients with one or two F508del alleles. ERJ Open Res. 2019; 5: 00082-02019.

8. Qiu F, Habgood M, Schneider-Futschik EK. The Balance between the Safety of Mother, Fetus, and Newborn Undergoing Cystic Fibrosis Transmembrane Conductance Regulator Treatments during Pregnancy. ACS Pharmacol. Transl. Sci. 2020; 3: 835-843.

9. Vekaria S, Popowicz N, White SW, et al. To be or not to be on CFTR modulators during pregnancy: Risks to be considered. J. Cyst. Fibros. 2020; 19: e7-e8.

10. Kaminski R, Nazareth D. A successful uncomplicated CF pregnancy while remaining on Ivacaftor. J. Cyst. Fibros. 2016; 15: 133-134.

11. Ladores S, Kazmerski TM, Rowe SM. A Case Report of Pregnancy During Use of Targeted Therapeutics for Cystic Fibrosis. J. Obstet. Gynecol. Neonatal Nurs. 2017; 46: 72-77.

12. Ladores S, Bray LA, Brown J. Two Unanticipated Pregnancies While on Cystic Fibrosis GeneSpecific Drug Therapy. J. Patient Exp. 2020; 7: 4-7.

13. Trimble A, McKinzie $\mathrm{C}$, Terrell $\mathrm{M}$, et al. Measured fetal and neonatal exposure to Lumacaftor and Ivacaftor during pregnancy and while breastfeeding. J. Cyst. Fibros. 2018; 17: 779-782.

14. Mainz JG, MichI RK, Beiersdorf N, et al. Successful Pregnancy of a Patient with Cystic Fibrosis Genotype F508del/F508del and Progressed Pulmonary Destruction on lumacaftor/ivacaftor. Klin. Pädiatr. 2019; 231: 271-273.

15. Jain R, Taylor-Cousar JL. Fertility, Pregnancy and Lactation Considerations for Women with CF in the CFTR Modulator Era. J. Pers. Med. 2021; 11: 418.

16. Tizzano EF, O'Brodovich $\mathrm{H}$, Chitayat $\mathrm{D}$, et al. Regional expression of CFTR in developing human respiratory tissues. Am. J. Respir. Cell Mol. Biol. American Thoracic Society - AJRCMB; 1994; 10: 355-362. 
17. Brennan SC, Wilkinson WJ, Tseng H-E, et al. The extracellular calcium-sensing receptor regulates human fetal lung development via CFTR. Sci. Rep. 2016; 6: 21975.

18. Meyerholz DK, Stoltz DA, Gansemer ND, et al. Lack of cystic fibrosis transmembrane conductance regulator disrupts fetal airway development in pigs. Lab. Invest. 2018; 98: 825838.

19. Qiu F, Habgood MD, Huang Y, et al. Entry of cystic fibrosis transmembrane conductance potentiator ivacaftor into the developing brain and lung. J. Cyst. Fibros. 2021; 20(5):857-864.

20. Van Doorninck JH, French PJ, Verbeek E, et al. A mouse model for the cystic fibrosis AF508 mutation. : 9 .

21. Del Moral P-M, Warburton D. Explant Culture of Mouse Embryonic Whole Lung, Isolated Epithelium, or Mesenchyme Under Chemically Defined Conditions as a System to Evaluate the Molecular Mechanism of Branching Morphogenesis and Cellular Differentiation. In: Ward A, Tosh D, editors. Mouse Cell Cult. [Internet] Totowa, NJ: Humana Press; 2010 [cited 2021 Jul 29]. p. 71-79Available from: http://link.springer.com/10.1007/978-1-59745-019-5_5.

22. Boucherat $\mathrm{O}$, Nadeau V, Bérubé-Simard F-A, et al. Crucial requirement of ERK/MAPK signaling in respiratory tract development. Development 2015; 142: 3801-3801.

23. Bellusci S, Grindley J, Emoto H, et al. Fibroblast growth factor 10 (FGF10) and branching morphogenesis in the embryonic mouse lung. Development 1997; 124: 4867-4878.

24. Chuang P-T, Kawcak T, McMahon AP. Feedback control of mammalian Hedgehog signaling by the Hedgehog-binding protein, Hip1, modulates Fgf signaling during branching morphogenesis of the lung. Genes Dev. 2003; 17: 342-347.

25. Harris A, Chalkley G, Goodman S, et al. Expression of the cystic fibrosis gene in human development. P F 1983; : 8 .

26. McCray PB, Reenstra WW, Louie E, et al. Expression of CFTR and presence of CAMP-mediated fluid secretion in human fetal lung. Am. J. Physiol.-Lung Cell. Mol. Physiol. American Physiological Society; 1992; 262: L472-L481.

27. Trezise AEO, Chambers JA, Wardle $\mathrm{CJ}$, et al. Expression of the cystic fibrosis gene in human foetal tissues. Hum. Mol. Genet. 1993; 2: 213-218.

28. Marcorelles P, Montier T, Gillet D, et al. Evolution of CFTR protein distribution in lung tissue from normal and CF human fetuses. Pediatr. Pulmonol. 2007; 42: 1032-1040.

29. Cohen JC, Larson JE, Killeen E, et al. CFTR and Wnt/beta-catenin signaling in lung development. BMC Dev. Biol. 2008; 8: 70.

30. Viart V, Bergougnoux A, Bonini J, et al. Transcription factors and miRNAs that regulate fetal to adult CFTR expression change are new targets for cystic fibrosis. Eur. Respir. J. 2015; 45: 116128.

31. Broackes-Carter FC. Temporal regulation of CFTR expression during ovine lung development: implications for CF gene therapy. Hum. Mol. Genet. 2002; 11: 125-131. 
32. Li H, Yang W, Mendes F, et al. Impact of the cystic fibrosis mutation F508del-CFTR on renal cyst formation and growth. Am. J. Physiol.-Ren. Physiol. 2012; 303: F1176-F1186.

33. Bergmann C, Guay-Woodford LM, Harris PC, et al. Polycystic kidney disease. Nat. Rev. Dis. Primer 2018; 4: 50.

34. Danopoulos S, Thornton ME, Grubbs BH, et al. Discordant roles for FGF ligands in lung branching morphogenesis between human and mouse. J. Pathol. 2018; path.5188.

35. Boucherat $\mathrm{O}$, Landry-Truchon $\mathrm{K}$, Bérubé-Simard F-A, et al. Epithelial inactivation of $Y y 1$ abrogates lung branching morphogenesis. Development 2015; 142: 2981-2995.

36. https://www.accessdata.fda.gov/drugsatfda_docs/label/2019/212273s000lbl.pdf).

37. Sun X, Yi Y, Yan Z, et al. In utero and postnatal VX-770 administration rescues multiorgan disease in a ferret model of cystic fibrosis. Sci. Transl. Med. 2019; 11: eaau7531.

38. Itoh N. FGF10: A multifunctional mesenchymal-epithelial signaling growth factor in development, health, and disease. Cytokine Growth Factor Rev. 2016; 28: 63-69. 


\section{Figure legends}

\section{Figure 1. Ex vivo lung explants from E12.5 $\mathrm{Cftr}^{\text {tm1Eur }}$ mouse embryos.}

A. Lung explants from WT $(A-D)(n=12), C^{t t m 1 E u r /+}(E-H)(n=31)$, and Cftr $^{\mathrm{tm} 1 \text { Eur/tm1Eur }}(I-$ $L)(n=16)$ embryos cultured for 3 days in DMEM/F12. Scale bar: $1 \mathrm{~mm}$.

B. Number of terminal buds at T24, T48 and T72, expressed as the percentage in branching increase compared to baseline (TO).

C. Number of terminal buds' dilations at T72, expressed as the percentage of the dilations increase compared to mean number of terminal buds' dilations in the media group.

Data are expressed as mean \pm s.e.m.

Figure 2. Ex vivo lung explants from E12.5 $\mathrm{Cftr}^{\mathrm{tm} 1 \mathrm{Eur} /+}$ embryos cultured with Elexacaftor/Tezacaftor/lvacaftor association.

A. Lung explants from Cftr $^{\text {tm1Eur/+ }}$ embryos cultured for 3 days in DMEM/F12 alone (AD) $(n=31)$, or DMEM/F12+ETI $(E-H)(n=9)$. Scale bar: $1 \mathrm{~mm}$.

B. Number of terminal buds at T24, T48 and T72, expressed as the percentage in branching increase compared to baseline (TO).

C. Number of terminal buds' dilations at T72, expressed as the percentage of the dilations increase compared to mean number of terminal buds' dilations in the media group.

Data are expressed as mean \pm s.e.m. ${ }^{* * *} \mathrm{p}<0.0001$. 
Figure 3. Ex vivo lung explants from E12.5 WT mouse embryos cultured with Elexacaftor/Tezacaftor/Ivacaftor association.

A. Lung explants from WT embryos cultured for 3 days in DMEM/F12 alone (AD) $(n=9)$, or DMEM/F12+ETI $(E-H)(n=6)$. Scale bar: $1 \mathrm{~mm}$.

B. Number of terminal buds at T24, T48 and T72, expressed as the percentage in branching increase compared to baseline (T0).

C. Number of terminal buds' dilations at T72, expressed as the percentage of the dilations increase compared to mean number of terminal buds' dilations in the media group.

Data are expressed as mean \pm s.e.m. ${ }^{* * *} p<0.001,{ }^{* * * *} p<0.0001$.

Figure 4. Ex vivo lung explants from E12.5 WT mouse embryos cultured with Forskolin or Forskolin+Inh172

A. Lung explants from WT embryos cultured for 3 days in DMEM/F12 alone (AD) $(n=9), \quad$ DMEM/F12+Forskolin $(E-H)(n=8)$, or DMEM/F12 +Forskolin+Inh172 (I$\mathrm{L})(\mathrm{n}=5)$. Scale bar: $1 \mathrm{~mm}$.

B. Number of terminal buds at T24, T48 and T72, expressed as the percentage in branching increase compared to baseline (T0).

C. Number of terminal buds' dilations at T72, expressed as the percentage of the dilations increase compared to mean number of terminal buds' dilations in the media group.

Data are expressed as mean \pm s.e.m. ${ }^{*} p<0.05,{ }^{* *} p<0.01,{ }^{* * *} p<0.001,{ }^{* * *} p<0.0001$ 
Figure 5. RT-qPCR gene expression of Fgf10, Fgfr2IIIb, Shh and Hhip in mice embryonic lung explant

A. Explants from $\mathrm{Cftr}^{\mathrm{tm} 1 \mathrm{Eur} /+}$ embryos cultured for 3 days in DMEM/F12 alone $(\mathrm{n}=5)$, or DMEM/F12+ETI (n=6).

B. Explants from WT embryos cultured for 3 days in DMEM/F12 alone $(n=5)$, DMEM/F12+ETI $\quad(n=5), \quad$ DMEM/F12+Forskolin $\quad(n=3), \quad$ or $\quad$ DMEM/F12 +Forskolin+Inh172 ( $\mathrm{n}=4)$.

Data are expressed as mean \pm s.e.m. ${ }^{*} p<0.05,{ }^{* *} p<0.01$.

Figure S1. RT-qPCR Cftr expression during murine lung development mRNA Cftr expression in lung from WT embryos (E12.5, E15.5 and E18.5) and newborns (P0, P7, P15 and P30).

Data are expressed as mean \pm s.e.m. ${ }^{* *} \mathrm{p}<0.01,{ }^{* * * *} \mathrm{p}<0,0001$

Figure S2. Ex vivo lung explants from E12.5 WT mouse embryos cultured with different drugs' vehicle

A. Lung explants from WT embryos cultured for 3 days in DMEM/F12 alone (AD) $(n=9)$, DMEM/F12+DMSO $(E-H)(n=15)$, or DMEM/F12+EtOH $(I-L)(n=12)$. Scale bar: $1 \mathrm{~mm}$.

B. Number of terminal buds at T24, T48 and T72, expressed as the percentage in branching increase compared to baseline (T0).

C. Number of terminal buds' dilations at T72, expressed as the percentage of the dilations increase compared to mean number of terminal buds' dilations in the media group.

Data are expressed as mean \pm s.e.m. ${ }^{* *} \mathrm{p}<0.01,{ }^{* * * *} \mathrm{p}<0,0001$ 


\section{Figure S3. Ex vivo lung explants from E12.5 WT mouse embryos cultured with}

\section{Inh172}

A. Lung explants from WT embryos cultured for 3 days in DMEM/F12 alone (A-

D) $(n=9)$, or DMEM/F12+Inh172 $(E-H)(n=14)$. Scale bar: $1 \mathrm{~mm}$.

B. Number of terminal buds at T24, T48 and T72, expressed as the percentage in branching increase compared to baseline (T0).

C. Number of terminal buds' dilations at T72, expressed as the percentage of the dilations increase compared to mean number of terminal buds' dilations in the media group.

Data are expressed as mean \pm s.e.m. 
Table 1. Lung branching and terminal buds' dilations in WT Cftr $^{+/+}$and F508del heterozygous Cftr ${ }^{\mathrm{tm} 1 \mathrm{Eur} /+}$ lung explant culture

\begin{tabular}{|c|c|c|c|c|c|c|c|c|c|c|c|c|c|}
\hline \multirow{3}{*}{ Experiment } & \multirow{3}{*}{ Treatment } & \multicolumn{9}{|c|}{ Branching increase (\%) } & \multirow{2}{*}{\multicolumn{3}{|c|}{$\begin{array}{c}\text { Terminal buds' dilations } \\
\text { at T72 (\%) }\end{array}$}} \\
\hline & & \multicolumn{3}{|c|}{ T24 } & \multicolumn{3}{|c|}{$\mathrm{T} 48$} & \multicolumn{3}{|c|}{$\mathrm{T} 72$} & & & \\
\hline & & $\begin{array}{c}\text { mean } \\
\text { (s.e.m) }\end{array}$ & $\begin{array}{r}p-v \\
\text { T24 v }\end{array}$ & & $\begin{array}{c}\text { mean } \\
\text { (s.e.m) }\end{array}$ & $\begin{aligned} p-1 \\
\text { T48 }\end{aligned}$ & $\begin{array}{l}\text { alue } \\
\text { vs T0 }\end{array}$ & $\begin{array}{c}\text { mean } \\
\text { (s.e.m) }\end{array}$ & $\begin{array}{r}\mathrm{p}- \\
\mathrm{T72}\end{array}$ & $\begin{array}{l}\text { alue } \\
\text { vs T0 }\end{array}$ & $\begin{array}{c}\text { mean } \\
\text { (s.e.m) }\end{array}$ & $p-1$ & alue \\
\hline \multirow{3}{*}{$\begin{array}{l}\text { Cftr }^{\text {tm1Eur }} \\
\text { lung } \\
\text { explant } \\
\text { culture }\end{array}$} & $\begin{array}{c}\text { Cftr }^{+/+} \\
(n=13)\end{array}$ & $\begin{array}{c}71.0 \\
(10.2)\end{array}$ & & & $\begin{array}{c}196.4 \\
(18.91)\end{array}$ & & & $\begin{array}{l}301.9 \\
(27.2)\end{array}$ & & & $\begin{array}{l}21.8 \\
(2.8)\end{array}$ & & \\
\hline & $\begin{array}{c}\text { Cftr }^{\mathrm{tm} 1 \text { Eurr/+ }} \\
(\mathrm{n}=31)\end{array}$ & $\begin{array}{l}70.1 \\
(5.3)\end{array}$ & \multicolumn{2}{|c|}{$N S^{a}$} & $\begin{array}{l}190.0 \\
(13.1)\end{array}$ & \multicolumn{2}{|c|}{$N S^{a}$} & $\begin{array}{l}263.4 \\
(15.2)\end{array}$ & \multicolumn{2}{|c|}{$N S^{a}$} & $\begin{array}{l}14.6 \\
(1.7)\end{array}$ & \multicolumn{2}{|c|}{$N S^{a}$} \\
\hline & $\begin{array}{c}C f t r^{\mathrm{tm} 1 \text { Eur/tm1Eur }} \\
(\mathrm{n}=16)\end{array}$ & $\begin{array}{l}67.9 \\
(7.8)\end{array}$ & $\mathrm{NS}^{\mathrm{a}}$ & $N S^{b}$ & $\begin{array}{c}171.4 \\
(16.71) \\
\end{array}$ & $N S^{a}$ & $N S^{b}$ & $\begin{array}{l}281.0 \\
(20.16) \\
\end{array}$ & $N S^{a}$ & $N S^{b}$ & $\begin{array}{l}18.8 \\
(3.2) \\
\end{array}$ & $N S^{a}$ & $N S^{b}$ \\
\hline \multirow{2}{*}{$\begin{array}{l}\text { Cftr }{ }^{\mathrm{tm} 1 \mathrm{Eur} /+} \\
\text { lung } \\
\text { explant } \\
\text { culture }\end{array}$} & $\begin{array}{c}\text { DMEM/F12 } \\
(\mathrm{n}=31)\end{array}$ & $\begin{array}{l}70.1 \\
(5.3)\end{array}$ & \multirow{2}{*}{\multicolumn{2}{|c|}{$N S^{c}$}} & $\begin{array}{c}190.0 \\
(13.14)\end{array}$ & & & $\begin{array}{l}263.4 \\
(15.2)\end{array}$ & & & $\begin{array}{l}14.6 \\
(1.7)\end{array}$ & & \\
\hline & $\begin{array}{c}\text { ETI } \\
(n=9)\end{array}$ & $\begin{array}{l}34.0 \\
(5.7) \\
\end{array}$ & & & $53.0(5.1)$ & $<0$ & $001^{c}$ & $\begin{array}{l}57.8 \\
(6.0)\end{array}$ & $<0$ & $001^{c}$ & $\begin{array}{l}76.9 \\
(4.7) \\
\end{array}$ & $<0$. & $001^{c}$ \\
\hline \multirow{4}{*}{$\begin{array}{c}\text { Cftr }^{+/+} \text {lung } \\
\text { explant } \\
\text { culture }\end{array}$} & $\begin{array}{c}\text { DMEM/F12 } \\
(n=9)\end{array}$ & $\begin{array}{l}76.7 \\
(5.0)\end{array}$ & & & $\begin{array}{c}218.7 \\
(8.2)\end{array}$ & & & $\begin{array}{c}305.5 \\
(8.4)\end{array}$ & & & $\begin{array}{l}12.3 \\
(5.0)\end{array}$ & & \\
\hline & $\begin{array}{c}E T I \\
(n=6)\end{array}$ & $\begin{array}{l}46.5 \\
(9.1)\end{array}$ & \multicolumn{2}{|c|}{$N S^{c}$} & $\begin{array}{c}55.7 \\
(12.3)\end{array}$ & $<0$ & $001^{c}$ & $\begin{array}{c}63.2 \\
(11.3)\end{array}$ & \multicolumn{2}{|c|}{$<0.0001^{\mathrm{c}}$} & $\begin{array}{l}70.3 \\
(3.9)\end{array}$ & \multicolumn{2}{|c|}{$0.0004^{c}$} \\
\hline & $\begin{array}{c}\text { Forskolin } \\
(n=8)\end{array}$ & $\begin{array}{l}25.1 \\
(2.8)\end{array}$ & \multicolumn{2}{|c|}{$0.0001^{c}$} & $\begin{array}{l}139.6 \\
(9.0)\end{array}$ & $<0$. & $001^{c}$ & $\begin{array}{l}188.0 \\
(11.7) \\
\end{array}$ & \multicolumn{2}{|c|}{$<0.0001^{\mathrm{c}}$} & $\begin{array}{l}28.3 \\
(4.1)\end{array}$ & \multicolumn{2}{|c|}{$0.0492^{c}$} \\
\hline & $\begin{array}{c}\text { Forskolin+Inh172 } \\
(\mathrm{n}=5)\end{array}$ & $\begin{array}{l}61.8 \\
(8.1)\end{array}$ & $N^{c}$ & $N S^{d}$ & $\begin{array}{l}213.1 \\
(25.2)\end{array}$ & $N S^{c}$ & $0.0007^{d}$ & $\begin{array}{l}292.3 \\
(16.9)\end{array}$ & $N S^{c}$ & $<0.0001^{d}$ & $\begin{array}{c}4.1 \\
(2.0)\end{array}$ & $N^{c}$ & $0.0016^{d}$ \\
\hline
\end{tabular}

Mean \pm s.e.m. value and statistical analysis of branching increase (2way ANOVA) and terminal buds' dilations (Mann-Withney test).

a: versus $\mathrm{Cftr}^{+/+}$. b: versus Cftr $^{\text {tm1Eur/+ }}$. c: versus DMEM/F12. d: versus Forskolin. NS: not significant. 
Table 2. Fgf10, Fgfr2IIlb, Shh and Hhip expression in in WT Cftr ${ }^{+/+}$and F508del heterozygous Cftr $^{\text {tm1Eur/+ }}$ lung explant

\begin{tabular}{|c|c|c|c|c|c|c|c|c|c|c|c|c|}
\hline \multirow{3}{*}{ Experiment } & \multirow{3}{*}{ Treatment } & \multicolumn{11}{|c|}{ gene expression (\%) } \\
\hline & & \multicolumn{3}{|c|}{ Fgf10 } & \multicolumn{3}{|c|}{ Fgfr2IIIb } & \multicolumn{3}{|c|}{ Shh } & \multicolumn{2}{|c|}{ Hhip } \\
\hline & & $\begin{array}{l}\text { mean } \\
\text { (s.e.m) }\end{array}$ & \multicolumn{2}{|c|}{$p$-value } & $\begin{array}{l}\text { mean } \\
\text { (s.e.m) }\end{array}$ & \multicolumn{2}{|c|}{$p$-value } & $\begin{array}{l}\text { mean } \\
\text { (s.e.m) }\end{array}$ & \multicolumn{2}{|c|}{$p$-value } & $\begin{array}{l}\text { mean } \\
\text { (s.e.m) }\end{array}$ & p-valu㶨 \\
\hline \multirow{2}{*}{$\begin{array}{c}\text { Cftr }^{\text {tmikur/+ }} \\
\text { lung } \\
\text { explant } \\
\text { culture } \\
\end{array}$} & DMEM/F12 $(n=5)$ & $\begin{array}{l}100.0 \\
(12.1)\end{array}$ & & & $\begin{array}{l}100.0 \\
(11.7)\end{array}$ & & & $100.0(3.9)$ & & & $\begin{array}{l}100.0 \\
(18.7)\end{array}$ & \\
\hline & ETI $(n=6)$ & $\begin{array}{c}39.6 \\
(12.8)\end{array}$ & \multicolumn{2}{|c|}{$0.0173^{\mathrm{a}}$} & $5.7(1.5)$ & \multicolumn{2}{|c|}{$0.0043^{\mathrm{a}}$} & $36.9(9.8)$ & \multicolumn{2}{|c|}{$0.0043^{a}$} & $26.4(8.3)$ & $0.0043^{\frac{0}{2}}$ \\
\hline \multirow{4}{*}{$\begin{array}{l}\mathrm{Cftr}^{+/+} \text {lung } \\
\text { explant } \\
\text { culture }\end{array}$} & DMEM/F12 (n=5) & $\begin{array}{l}100.0 \\
(3.6)\end{array}$ & & & $100.0(4.4)$ & & & $100.0(5.1)$ & & & $100.0(6.7)$ & \\
\hline & $\operatorname{ETI}(n=5)$ & $\begin{array}{c}49.5 \\
(10.0)\end{array}$ & \multicolumn{2}{|c|}{$0.0079^{a}$} & $26.2(8.7)$ & \multicolumn{2}{|c|}{$0.0079^{a}$} & $42.0(6.7)$ & \multicolumn{2}{|c|}{$0.0079^{a}$} & $26.2(7.0)$ & $0.0079^{\frac{1}{3}}$ \\
\hline & Forskolin $(n=3)$ & $41.6(4.0)$ & \multicolumn{2}{|c|}{$0.0357^{\mathrm{a}}$} & $\begin{array}{l}244.4 \\
(56.1)\end{array}$ & \multicolumn{2}{|c|}{$0.0357^{\mathrm{a}}$} & $\begin{array}{l}210.0 \\
(37.1)\end{array}$ & \multicolumn{2}{|c|}{$0.0357^{\mathrm{a}}$} & $48.8(2.9)$ & 0.035 裹 \\
\hline & $\begin{array}{c}\text { Forskolin+lnh172 } \\
(n=4)\end{array}$ & $87.5(5.9)$ & $N S^{a}$ & $N S^{b}$ & $149.3(5.4)$ & 0.0159 & $N S^{b}$ & $\begin{array}{l}123.8 \\
(10.9) \\
\end{array}$ & $N S^{a}$ & $N S^{b}$ & $82.6(10.0)$ & $N S^{a}$ \\
\hline
\end{tabular}

Mean \pm s.e.m. value and statistical analysis by Mann-Withney test.

a: versus DMEM/F12. b: versus Forskolin. NS: not significant. 
Table S1: Primers sequences used RT-qPCR analysis

\begin{tabular}{|c|c|c|}
\hline Gene & Sequence (5'-3') & $\begin{array}{c}\text { Fragment } \\
\text { size (bp) }\end{array}$ \\
\hline Gapdh & $\begin{array}{c}\text { F - CTGCACCACCAACTGCTTAG } \\
\text { R - CCATCCACAGTCTTCTGGGT }\end{array}$ & 117 \\
\hline Rp/19 & $\begin{array}{c}\text { F - GATCATCCGCAAGCCTGTGA } \\
\text { R - GCATCCGAGCATTGGCAGTA }\end{array}$ & 122 \\
\hline Cftr & $\begin{array}{c}\text { F - CTTGCGCTGGTTCCAAATGA } \\
\text { R - GCTCACAGATCGCATCAAGC }\end{array}$ & 199 \\
\hline Hprt1 & $\begin{array}{l}\text { F - CAGTACAGCCCCAAAATGGT } \\
\text { R - GTCTGGCCTGTATCCAACAC }\end{array}$ & 78 \\
\hline Fgfr2IIlb & $\begin{array}{c}\text { F - TCAAAGCCATCAACAGCAACTATT } \\
\text { R - GATCTTTCAGCTTACAGTCGTTGTTAAA }\end{array}$ & 95 \\
\hline Shh & $\begin{array}{c}\text { F - TGACTCAGAGGTGCAAAGACA } \\
\text { R - ACTCCTCTGAATGATGGCCG }\end{array}$ & 120 \\
\hline Hhip & $\begin{array}{c}\text { F - CACTTCAACAGCACCAACCA } \\
\text { R - AGTAGGATGTCGATCCACGG }\end{array}$ & 87 \\
\hline
\end{tabular}


Table S2. Mean value and statistical analysis of WT Cftr $^{+/+}$and F508del heterozygous Cftr $^{\mathrm{tm} 1 \text { Eur/+ }}$ lung explant culture upon DMEM/F12 DMSO, EtOH, Inh172

\begin{tabular}{|c|c|c|c|c|c|c|c|c|c|c|c|c|c|}
\hline \multirow{3}{*}{ Experiment } & \multirow{3}{*}{ Treatment } & \multicolumn{9}{|c|}{ Branching increase (\%) } & \multicolumn{3}{|c|}{ Terminal buds' dilations } \\
\hline & & \multicolumn{3}{|c|}{ T24 vs T0 } & \multicolumn{3}{|c|}{ T48 vs T0 } & \multicolumn{3}{|c|}{ T72 vs T0 } & & & \\
\hline & & $\begin{array}{c}\text { mean } \\
\text { (s.e.m) }\end{array}$ & \multicolumn{2}{|c|}{ p-value } & $\begin{array}{c}\text { mean } \\
\text { (s.e.m) }\end{array}$ & \multicolumn{2}{|c|}{ p-value } & $\begin{array}{c}\text { mean } \\
\text { (s.e.m) }\end{array}$ & \multicolumn{2}{|c|}{ p-value } & $\begin{array}{c}\text { mean } \\
\text { (s.e.m) }\end{array}$ & & value \\
\hline \multirow{4}{*}{$\begin{array}{c}\text { Cftr }^{+/+} \text {lung } \\
\text { explant } \\
\text { culture } \\
\text { (control } \\
\text { groups) }\end{array}$} & $\begin{array}{c}\text { DMEM/F12 } \\
(n=9)\end{array}$ & $\begin{array}{l}76.7 \\
(5.0)\end{array}$ & & & $\begin{array}{c}218.7 \\
(8.2)\end{array}$ & & & $\begin{array}{c}305.5 \\
(8.4)\end{array}$ & & & $\begin{array}{l}12.3 \\
(5.0)\end{array}$ & & \\
\hline & $\begin{array}{l}\text { DMSO } \\
(n=15)\end{array}$ & $\begin{array}{l}87.3 \\
(9.5)\end{array}$ & \multicolumn{2}{|c|}{$\mathrm{NS}^{\mathrm{a}}$} & $\begin{array}{l}184.6 \\
(15.6)\end{array}$ & \multicolumn{2}{|c|}{$N^{a}$} & $\begin{array}{l}274.5 \\
(18.2)\end{array}$ & \multicolumn{2}{|c|}{$\mathrm{NS}^{\mathrm{a}}$} & $\begin{array}{l}18.4 \\
(2.7)\end{array}$ & \multicolumn{2}{|c|}{$N S^{a}$} \\
\hline & $\begin{array}{c}\text { EtOH } \\
(n=12)\end{array}$ & $\begin{array}{c}92.5 \\
(10.2)\end{array}$ & $N S^{a}$ & $N S^{b}$ & $\begin{array}{l}247.1 \\
(17.7)\end{array}$ & $N S^{a}$ & $0.0215^{b}$ & $\begin{array}{l}332.7 \\
(19.9)\end{array}$ & $N^{a}$ & $0.0356^{b}$ & $\begin{array}{c}7.1 \\
(2.6)\end{array}$ & $\mathrm{NS}^{\mathrm{a}}$ & $0.004 \frac{1}{a}$ \\
\hline & $\begin{array}{c}\text { Inh172 } \\
(\mathrm{n}=14)\end{array}$ & $\begin{array}{l}69.5 \\
(3.6) \\
\end{array}$ & $N S^{a}$ & $N S^{b}$ & $\begin{array}{l}191.9 \\
(6.2) \\
\end{array}$ & $N S^{a}$ & $N S^{b}$ & $\begin{array}{l}280.6 \\
(13.6)\end{array}$ & $N^{a}$ & $N S^{b}$ & $\begin{array}{c}6.5 \\
(1.7)\end{array}$ & $\mathrm{NS}^{\mathrm{a}}$ & $0 \Omega$ \\
\hline
\end{tabular}

Mean \pm s.e.m. value and statistical analysis of branching increase (2way ANOVA) and terminal buds' dilations (Mann-Withney test).

a: versus DMEM/F12. b: versus DMSO. NS: not significant. 


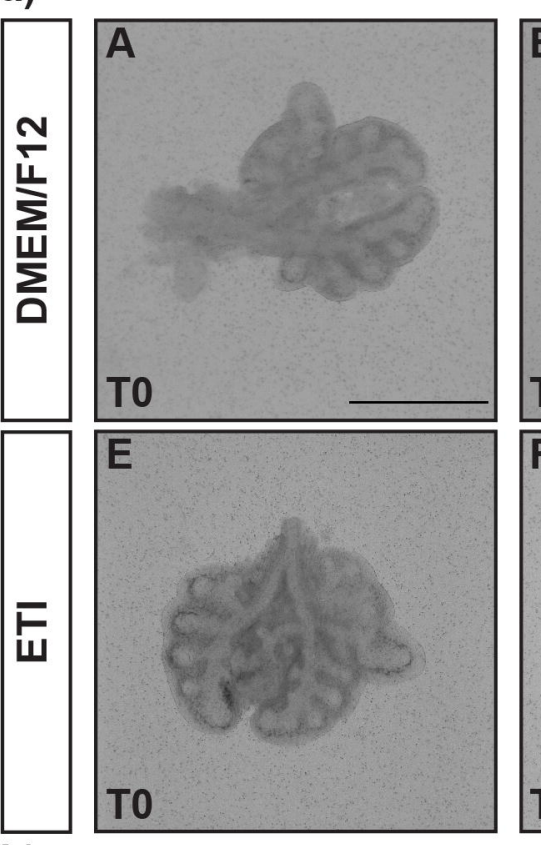

b)

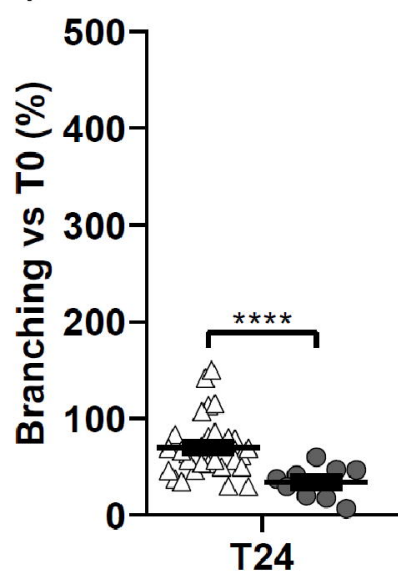

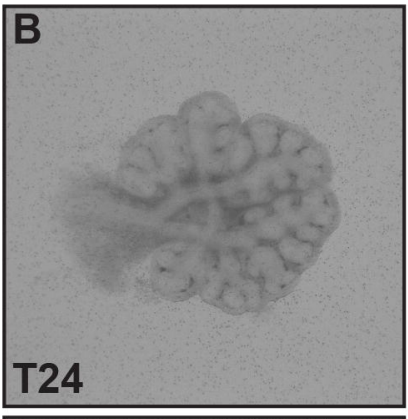

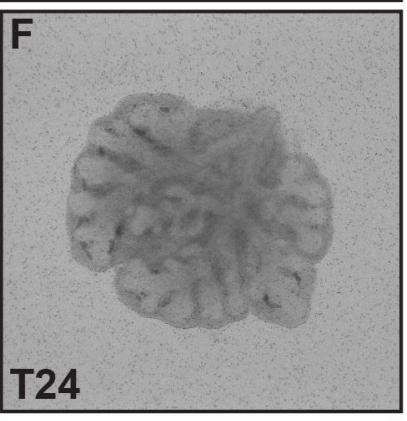

c)

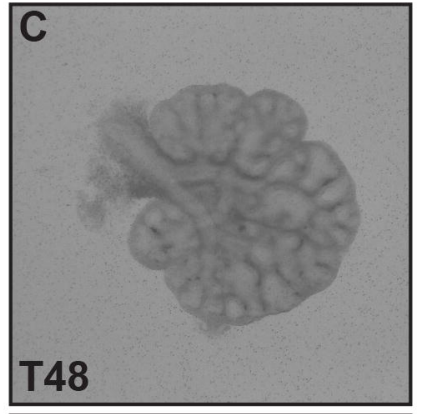

G

T48
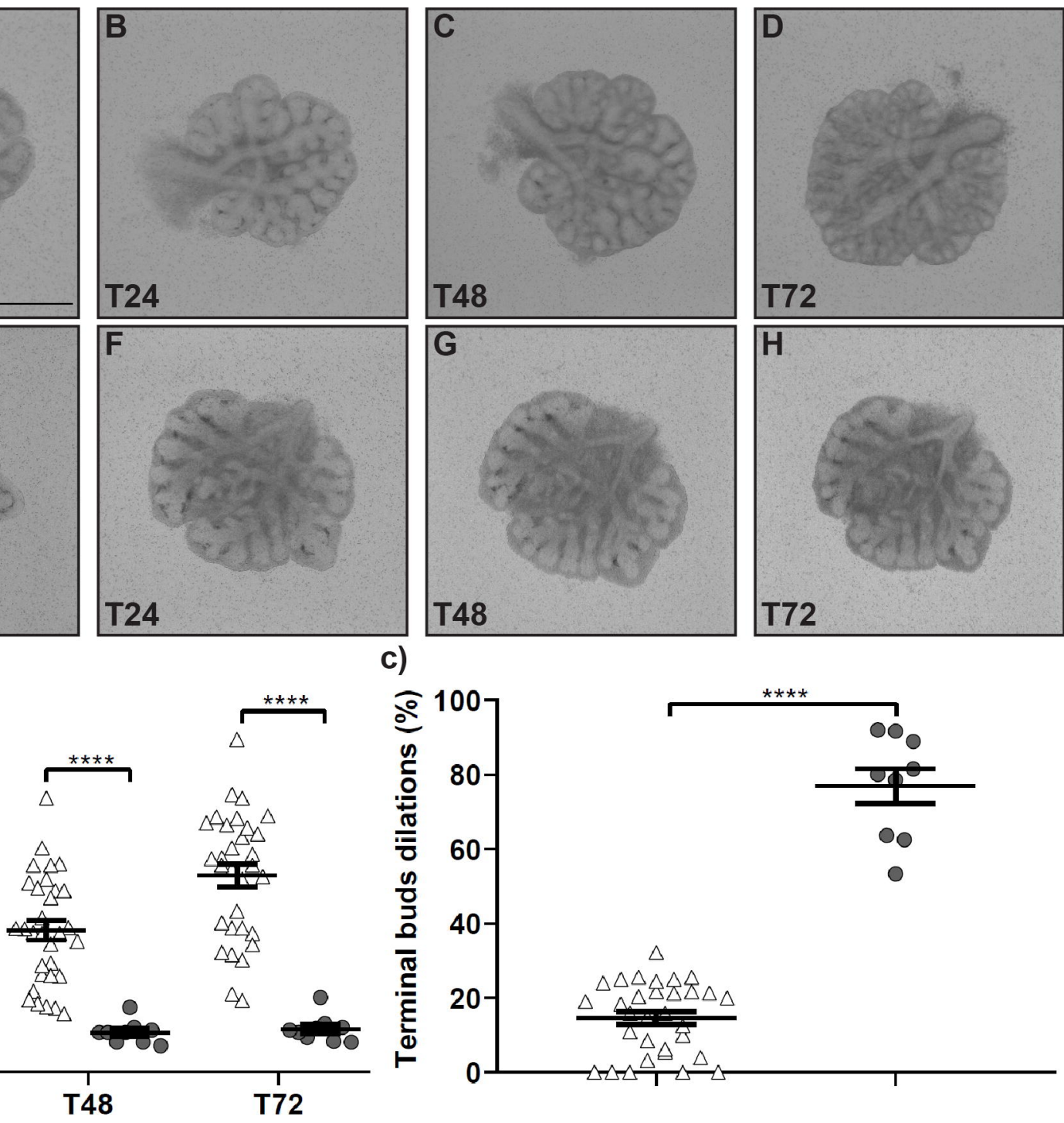

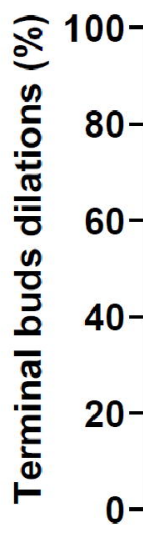

$\star * * *$

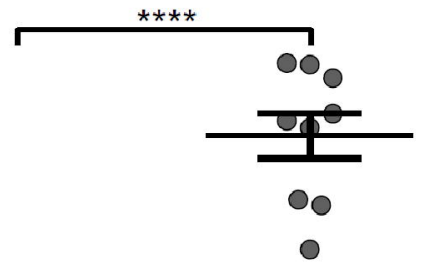

$\triangle \mathrm{DMEM} / \mathrm{F} 12 \bigcirc \mathrm{ETI}$ 


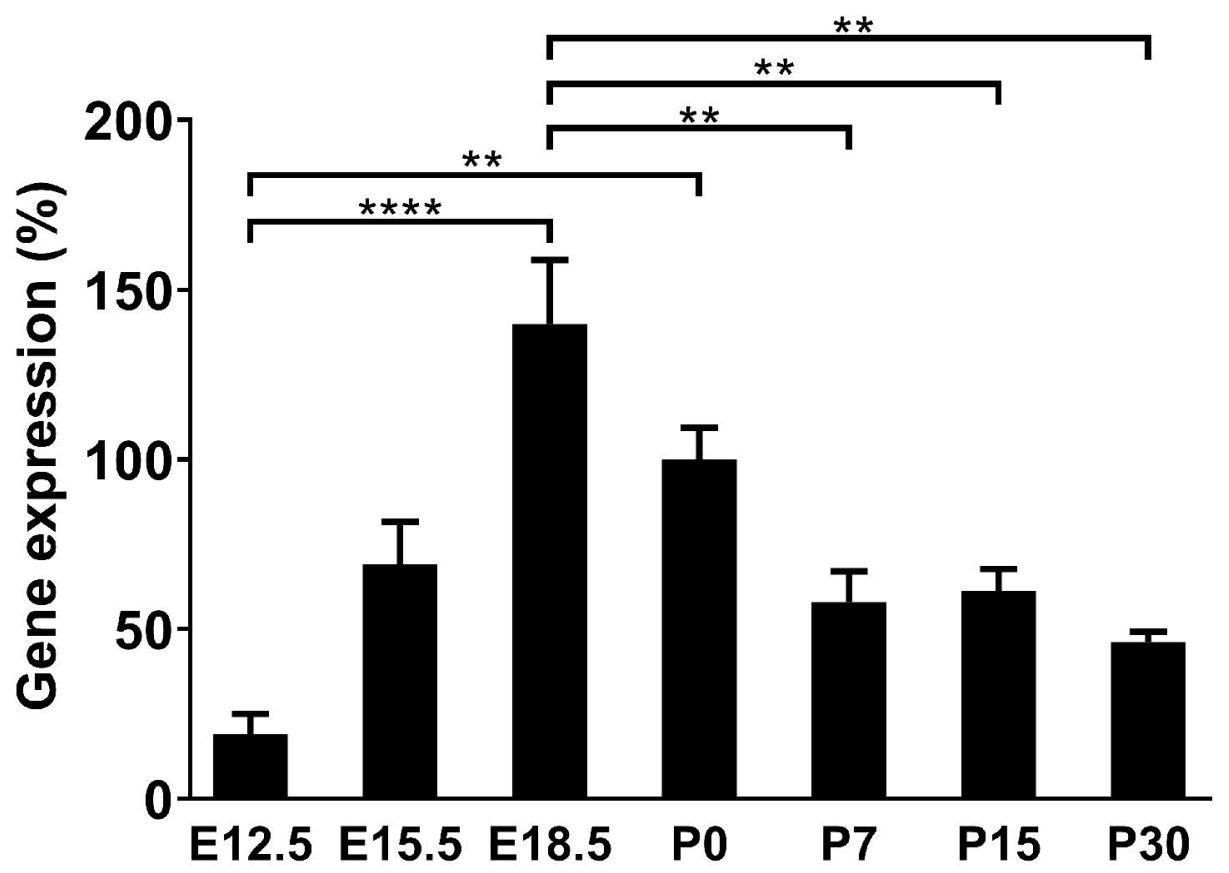

Figure $\mathbf{S 1}$ 
a)
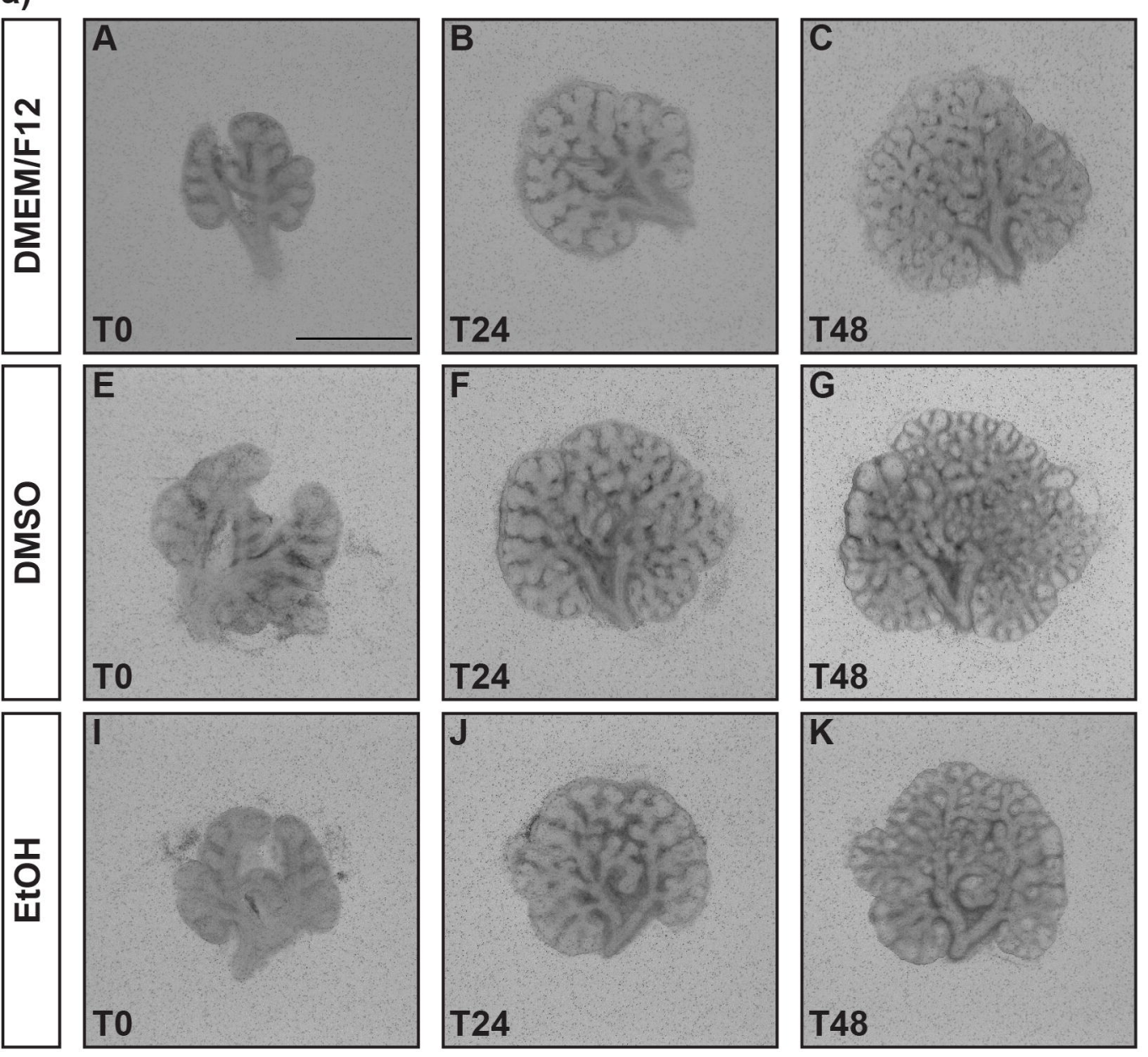

b)

(1)
TO

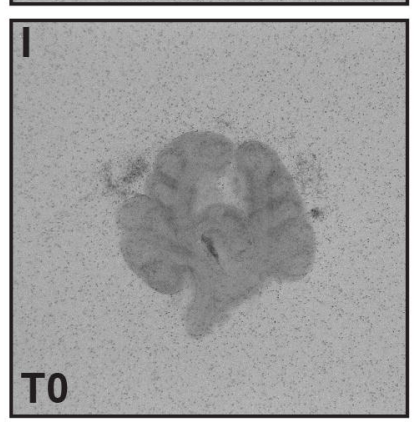

T24

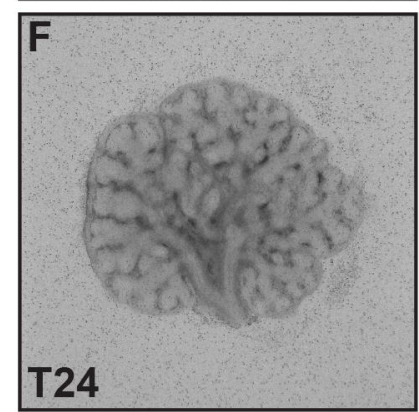

c)
T72

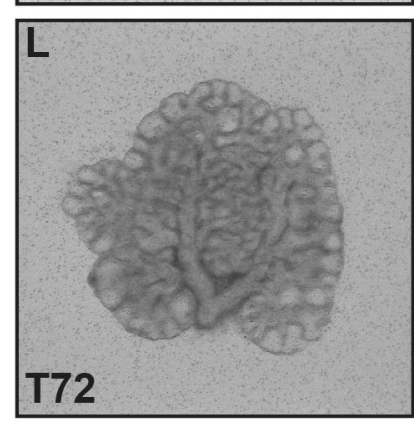

$\mathrm{T72}$
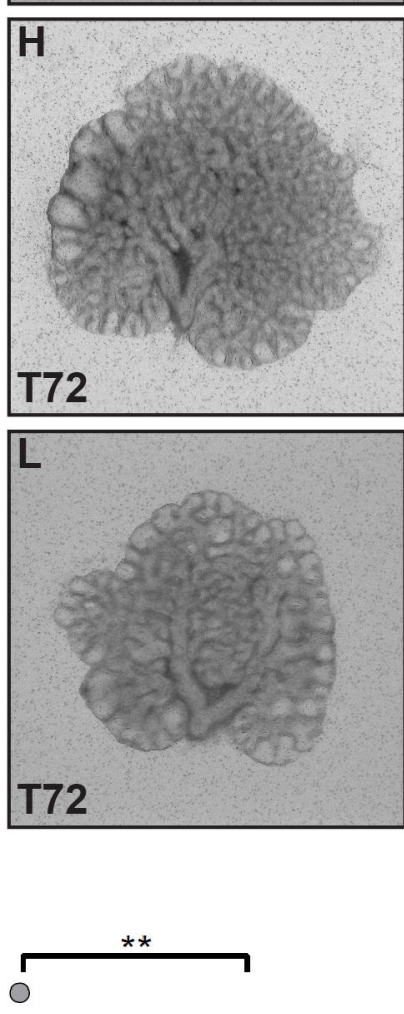

$\triangle$

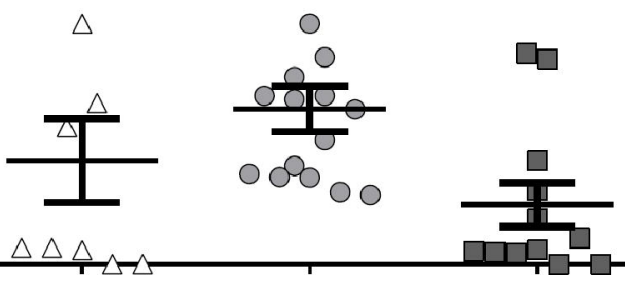

$\triangle$ DMEM/F12 $\quad$ DMSO $\square$ EtOH 
a)

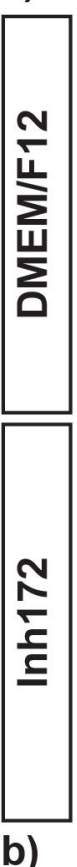

b)

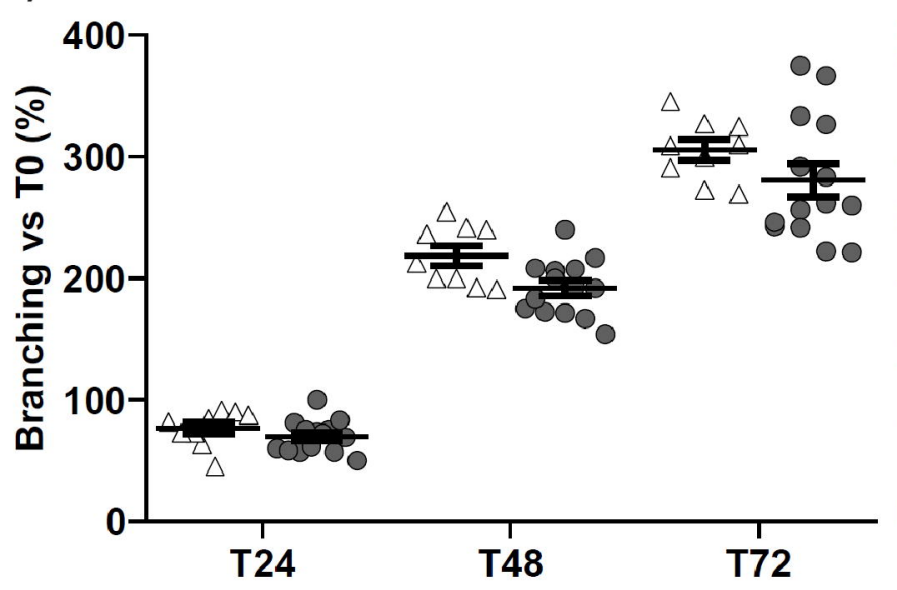

$\triangle$ DMEM/F12

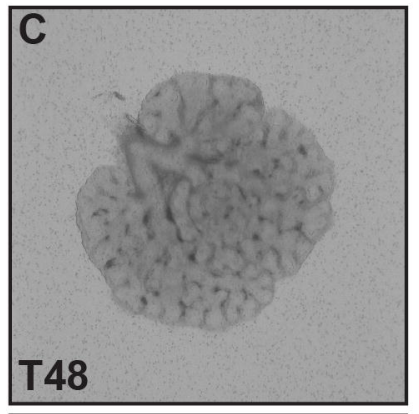

G

T48

c)
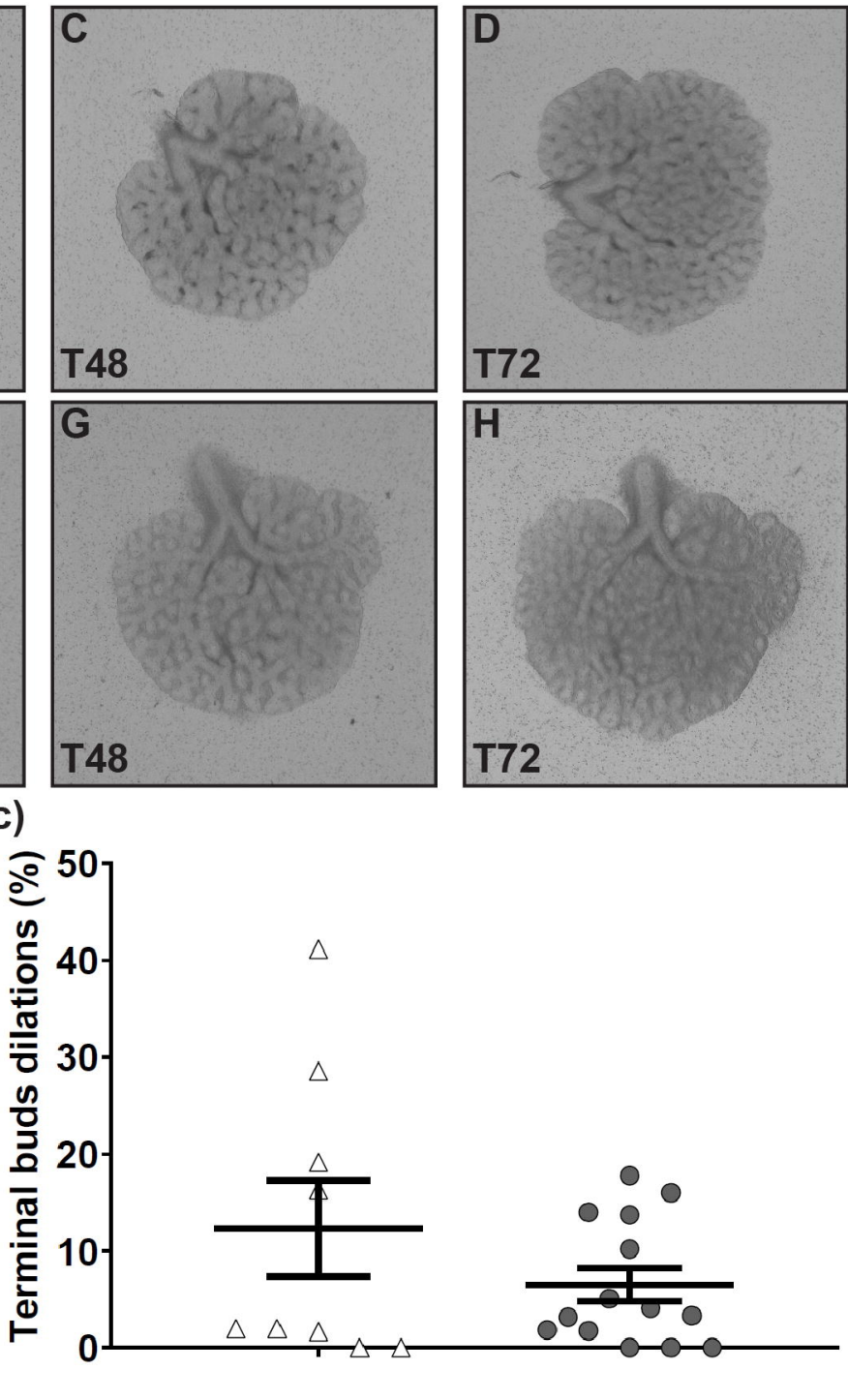

- Inh172 